\title{
State Estimator Condition Number Analysis
}

\author{
Reza Ebrahimian and Ross Baldick
}

\begin{abstract}
This paper develops formulas for the condition number of the state estimation problem as a function of the different types and number of measurements. We present empirical results using the IEEE RTS-96 and IEEE 118 bus systems that validate the formulas.
\end{abstract}

\section{INTRODUCTION}

TATE estimation in its original form is a least-squares $\checkmark$ problem. In a practical implementation, such a problem often may pose convergence problems. That is, it may converge slowly, produce inaccurate solutions, or be ill-conditioned to the point that it may never converge with finite precision arithmetic. Furthermore, as the number of buses in a system increases, the ill-conditioning of the state estimation problem becomes worse. Solution methods, such as orthogonal decomposition utilizing Givens rotations [1], [2], [9], [13], have been introduced to overcome this ill-conditioning without loss of sparsity.

A condition number analysis of the state estimation problem by $\mathrm{Gu}$, et al. [5] has shown that the type of measurements, for example branch flow or bus injection measurements, will affect the numerical conditioning of the problem. That paper showed that branch flow measurements are better than injection measurements for the numerical conditioning of the problem. Since in practical systems a mixture of voltage, flow, and injection measurements is used (and in the future, voltage angle measurements will become available [7], [8]), we extend the results of [5] by developing formulas to describe the dependence of the condition number on the mixtures of voltage, flow, and injection measurements. These formulas are approximations that provide good qualitative correspondence with the actual condition numbers produced by state estimation software.

In Section II we give a description of condition number; in Section III we present the assumptions and their effects on our analysis; in Section IV we develop analytical formulas for condition numbers when there are mixtures of different types and number of measurements; in Section $\mathrm{V}$ we extend our analyzes to qualitative descriptions of the condition numbers after orthogonal transformation; in Section VI we compare these analytical approximations with actual condition numbers for two IEEE test cases, using a state estimation software developed in MATLAB. We conclude in Section VII.

Manuscript received January 27, 2000; revised December 15, 2000. R. Baldick was supported in part by the National Science Foundation under Grant ECS-9457133.

The authors are with the University of Texas at Austin, TX 78712

Publisher Item Identifier S 0885-8950(01)03792-0.

\section{CONDITION NUMBER ANALYSIS}

The condition number of a nonsingular square matrix $A$ is defined as [6]:

$$
\operatorname{Cond}(A) \equiv\|A\| \times\left\|A^{-1}\right\|,
$$

where any matrix norm can be used. It quantifies the sensitivity of a system to changes in the data. A large condition number in (1) is indicative of an ill-conditioned matrix. The largeness of the condition number is related to available finite precision arithmetic. The more precision in the calculations, the higher the level of ill-conditioning that can be tolerated. For example for double precision arithmetic, a condition number of $10^{12}$ is indicative of a highly ill-conditioned matrix [10]. In other words for an observable system using double precision arithmetic, the condition number of the information matrix should be much less than $10^{12}$ to guarantee convergence. The condition number can also affect other functions, such as bad data detection.

In this analysis, to make the problem manageable, we use the 1-norm to calculate the condition number for the information matrix of the state estimation problem. Other norms such as 2-norm would yield qualitatively similar results, however it would make the theoretical analysis formidable.

\section{ASSUMPTIONS USED TO CLARIFY THE ANALYSIS}

To clarify our analysis we use the fast decoupled load flow assumptions [4], [12]. These assumptions give a good approximation to the power flow problem. To present a simplified analysis of the affect of different measurements, we:

1) approximate our information matrix utilizing the decoupled load flow assumption, which assumes that reactive power $Q$ does not vary with voltage angle and real power $P$ does not vary with voltage magnitude [4], [12],

2) assume that $\sin \left(\theta_{i}-\theta_{j}\right)$ is approximately equal to $\left(\theta_{i}-\right.$ $\theta_{j}$ ) where $\theta_{i}$ and $\theta_{j}$ are voltage angles of nodes $i$ and $j$ at two ends of a branch joining nodes $i$ and $j$,

3) assume that the reactance to resistance ratios of the branches are very large,

4) consider radial networks,

5) assume all branch admittance magnitudes are equal to $y_{L}$, all voltage error variances are equal to $R_{v}$, and all flow and injection measurement error variances are equal to $R_{p}$

Except for our assumptions on voltage and power measurement error variance values, these assumptions follow [5]. The assumptions on voltage and power measurements error variances slightly generalize [5]. In Sections VI-A and VI-B we will demonstrate that analysis based on a radial system provides good insights into the effects of mixtures of measurements in IEEE test case systems that include nonradial topology. 

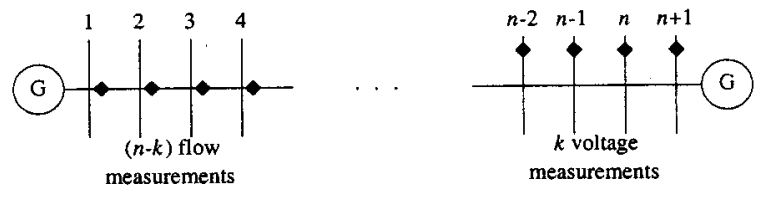

Fig. 1. Radial system with $n-k$ flow and $k$ voltage measurements.

Consider the maximum likelihood weighted least-squares state estimation problem:

$$
\min _{x} J(x)=\min \left[z^{\text {meas }}-f(x)\right]^{\dagger}\left[R^{-1}\right]\left[z^{\text {meas }}-f(x)\right],
$$

where,

$\begin{array}{ll}J(x) & \text { is the objective function, } \\ R & =\operatorname{diag}\left[\sigma^{2}\right], \\ \sigma^{2} & \text { is a vector of variances of the measurement } \\ & \text { errors, } \\ f & \text { is a vector of functions describing the } \\ & \text { measurements, } \\ z^{\text {meas }} & \text { is a vector of the measurements, } \\ x & \text { is a vector of the voltage magnitudes and } \\ & \text { angles, and }\end{array}$

the superscript $\dagger$ denotes transpose.

If the system is observable, then the Gauss-Newton update equations [14] for this nonlinear optimization problem are:

$$
\begin{aligned}
\Delta x^{(k)} & =\left[\begin{array}{c}
\Delta|E|^{(k)} \\
\Delta \theta^{(k)}
\end{array}\right] \\
& =\left[H^{\dagger} R^{-1} H\right]^{-1} H^{\dagger} R^{-1}\left[z^{\text {meas }}-f\left(x^{(k)}\right)\right], \\
x^{(k+1)} & =x^{(k)}+\Delta x^{(k)},
\end{aligned}
$$

where:

$H$
$R$

is the Jacobian of vector $f(x)$, is a diagonal matrix formed by the measurement error variances,

$|E|$ and $\theta$ are vectors of voltage magnitude and angles

superscript in indicates the iteration count, so that $x^{(k)}$ is parenthesis the value of iterate at the $k$ th iteration.

Now consider a radial system such as the one in Fig. 1. With fast decoupled load flow assumptions, the state estimator's Jacobian $H$ can be approximated as:

$$
H \approx\left[\begin{array}{cc}
H_{11} & 0 \\
0 & H_{22}
\end{array}\right],
$$

where: $H_{11}$ corresponds to voltage angle, real flow, and real injection measurements, and $H_{22}$ corresponds to voltage magnitude, reactive flow and reactive injection measurements.

With the above assumptions, we can write the state estimator information matrix as follows:

where,

$$
M^{\prime}=\left[\begin{array}{cc}
M_{11} & 0 \\
0 & M_{22}
\end{array}\right]
$$

$$
\begin{aligned}
& M^{\prime}=H^{\dagger} R^{-1} H, \\
& M_{11}=H_{11}^{\dagger} R_{11}^{-1} H_{11} \text { and } \\
& M_{22}=H_{22}^{\dagger} R_{22}^{-1} H_{22} .
\end{aligned}
$$

If for every real power measurement there is a corresponding reactive power measurement with equal measurement error variance, and for every voltage magnitude there is a corresponding voltage angle measurement, with equal measurement error variance then the information matrix $M_{11}$ is approximately the same as the information matrix $M_{22}$ and their condition numbers using 1-norm are both equal to the condition number of $M^{\prime}$. We have $M_{11} \approx M_{22}$, and for convenience we define, $M=M_{11}$.

In Section IV we develop equations for the condition number of the information matrix $M^{\prime}$ using the information matrix $M$. Hence, the condition numbers calculated in Section IV include the real and reactive flow and injection, as well as voltage magnitude and angle measurements.

We should emphasize that approximating the condition number of the information matrix $M^{\prime}$ utilizing the matrix $M$ is based on the assumptions listed earlier in this section. In particular the assumption concerning radial networks which makes these developments possible is a very rough approximation of looped networks presently in place.

In the calculation of the condition number of $H_{11}^{\dagger} R_{11}^{-1} H_{11}$, since branch admittance magnitude $y_{L}$ is assumed equal for all branches, $y_{L}^{2}$ can be factored out of the matrix $H_{11}^{\dagger} R_{11}^{-1} H_{11}$ and canceled out in the computation of its condition number. Therefore to clarify our presentation we set $y_{L}$ equal to one.

\section{EFFECTS OF COMBINATION OF DIFFERENT MEASUREMENTS ON THE CONDITION NUMBER}

In this section we develop formulas to describe the dependence of the condition number on the mixtures of voltage, flow, and injection measurements. We place a minimum number of measurements on radial networks, such that the system is observable. Thus, all of the measurements are critical, and removal of any measurement would render the system unobservable.

The results of [5] show the effects of flow-only measurements and injection-only measurements on the condition of the state estimation problem. Reference [5] demonstrates that flow measurements provided to the state estimator program yields much better condition number of the information matrix than injection measurements.

\section{A. Effects of Flow Measurements in the Presence of Voltage and Absence of Injection Measurements}

To assess the effect on the condition number of flow measurements in the presence of voltage and absence of injection measurements, we consider the system given in Fig. 1, for the case where there are $k$ voltage measurements on buses $n-k+2$ to bus $n+1$ and $n-k$ flow measurements on the branches starting from the branch after bus one to the branch before bus $n-k+1$.

For the system shown in Fig. 1 we calculate the Jacobian matrix $H$, given the Section III assumptions. Then the information matrix $M$ is:

$$
M=\left[\begin{array}{cc}
F / R_{p} & 0 \\
0 & V / R_{v}
\end{array}\right]
$$

where: $F$ is the information matrix given in [5] for the case of flow-only measurements, and $V$ is an identity matrix of size $k$. The inverse of the matrix in (6) is:

$$
M^{-1}=\left[\begin{array}{cc}
R_{p} F^{-1} & 0 \\
0 & R_{v} V^{-1}
\end{array}\right] .
$$



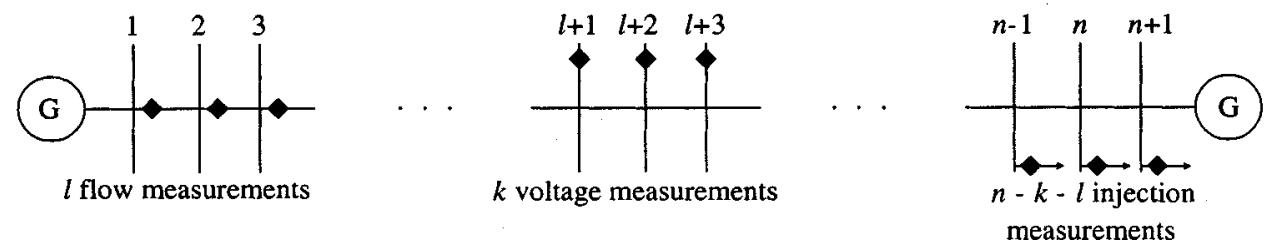

Fig. 2. Radial system with $l$ flow, $k$ voltage and $n-k-l$ injection measurements.

We can show that if:

$$
\frac{4}{R_{p}}<\frac{1}{R_{v}}
$$

then:

$$
\|M\|_{1}=\frac{1}{R_{v}}
$$

and if:

$$
R_{v}<\frac{R_{p}(n-k)(n-k+1)}{2},
$$

then we can show that:

$$
\left\|M^{-1}\right\|_{1}=\frac{R_{p}(n-k)(n-k+1)}{2} .
$$

The assumptions on $R_{p}$, and $R_{v}$ are reasonable. In summary, if $4 / R_{p}<1 / R_{v}$, and if $R_{v}<\left(R_{p}(n-k)(n-k+1)\right) / 2$, then the condition number is:

$$
C_{f v}^{M}=\frac{R_{p}(n-k)(n-k+1)}{2 R_{v}},
$$

for $n-k \geq 2$. It is obvious from (7) that for a given $n$, as the number of voltage measurements $k$ increases, the condition number decreases. Reference [5] arrives at the conclusion that the flow-only measurements will result in a condition number on the order of $n^{2}$. Here, we have shown that with $n-k$ flow and $k$ voltage measurements the condition number is on the order of $(n-k)^{2}$.

\section{B. Effects of Injection in the Presence of Voltage and Flow Measurements}

We place injection measurements on buses $k+l+2$ to $n+1$, flow measurements on branches starting from the branch after bus one to the branch before bus $l+1$, and we place voltage measurements on buses $l+2$ to $k+l+1$ as shown in Fig. 2. The resulting information matrix assuming equal error variances for all the measurements leads to a condition number which is independent of error variances [3]. However, with different values of error variances for power and voltage measurements the information matrix is:

$$
M=\left[\begin{array}{ccc}
F / R_{p} & 0 & 0 \\
0 & \hat{V} / R_{v} & 0 \\
0 & 0 & \hat{P} / R_{p}
\end{array}\right],
$$

where, the matrix $\hat{V}$ is an identity matrix of size $k-1$ and the matrix $\hat{P}$ is similar to the information matrix $P$ given in [5] for the case of injection-only measurements, except matrix $\hat{P}$ is augmented with an additional row and column of the following forms:

$$
\hat{P}_{1 i}=\left[\begin{array}{llllll}
\frac{R_{p}+R_{v}}{R_{v}} & -2 & 1 & 0 & \cdots & 0
\end{array}\right],
$$

and

$$
\hat{P}_{i 1}=\left[\begin{array}{llllll}
\frac{R_{p}+R_{v}}{R_{v}} & -2 & 1 & 0 & \cdots & 0
\end{array}\right]^{\dagger} .
$$

The inverse of the information matrix $M$ is:

$$
M^{-1}=\left[\begin{array}{ccc}
R_{p} F^{-1} & 0 & 0 \\
0 & R_{v} \hat{V}^{-1} & 0 \\
0 & 0 & R_{p} \hat{P}^{-1}
\end{array}\right] .
$$

The 1-norm of $M$ can be calculated from $\hat{P}$. If:

then we can show that:

$$
\frac{R_{p}+4 R_{v}}{R_{v}}>16
$$

$$
\|M\|_{1}=\frac{R_{p}+4 R_{v}}{R_{p} R_{v}} .
$$

This assumption on $R_{p}$ and $R_{v}$ is reasonable. If we let $j=$ $n-k-l$, then the 1 -norm of $M^{-1}$ can be calculated from $\hat{P}^{-1}$, and we can show that:

$$
\begin{aligned}
\left\|M^{-1}\right\|_{1}= & R_{p}\left\{\frac{j(j+1)\left[5 j^{2}+5 j+2\right]^{2}}{24}+j+1\right\} \\
& -(j+1)\left(R_{p}-R_{v}\right) .
\end{aligned}
$$

In summary, if $\left(R_{p}+4 R_{v}\right) / R_{v}>16$, and if we let $j=n-k-l$, then the condition number is:

$$
\begin{aligned}
& C_{i f v}^{M}=\frac{R_{p}+4 R_{v}}{R_{p} R_{v}}\left\{\frac{R_{p} j(j+1)\left[5 j^{2}+5 j+2\right]^{2}}{24}+R_{p}(j+1)\right. \\
& \left.-(j+1)\left(R_{p}-R_{v}\right)\right\},
\end{aligned}
$$

for: $j \geq 4$, and $k \geq 1$. The condition number is now on the order of $(n-k-l)^{4}$. We can conclude that the lower the proportion of injection measurements the better the condition of the information matrix, in the presence of voltage and flow measurements.

Now consider the same analysis in the absence of flow measurements; then we would have injection and voltage measurements. The information matrix in the absence of flow measurements using similar placements of measurements as given in Fig. 2 would be the same as of (8) except that the partition of the matrix corresponding to the flow measurements is removed. The inverse of the information matrix would be similar to (9) except that the first partition of the matrix (upper left hand) will be different. However the last partition (lower right hand), which is the partition used in the calculation of the condition number using the 1-norm, remains the same. The condition number $C_{i v}^{M}$ for this case therefore is the same as (10) where $l$ is set equal to zero, so that $j=n-k$.

This equation is written in terms of $j=n-k$ in order to demonstrate that the condition number is on the order of $(n-$ $k)^{4}$. In [5] the condition number for a case with injection-only measurements at all buses is $2 n(n+1)\left(5 n^{2}+5 n+2\right) / 3$. Notice that the condition number for the case of injection-only measurements increases as $n^{4}$ whereas the condition number 
with $k$ voltage measurements and $n-k$ injection measurements increases as $(n-k)^{4}$.

\section{ORTHOGONAL TRANSFORMATION AND THE CONDITION NUMBER}

Since, in practice, orthogonal transformation is used to improve the condition number of the matrices involved in the state estimation problem, we also present results for condition number analysis of these matrices. We can write the information matrix described in Section III as follows:

$$
H^{\dagger} R^{-1} H=\left[H^{\dagger} R^{-1 / 2} R^{-1 / 2} H\right]=\tilde{H}^{\dagger} \tilde{H},
$$

where: $\tilde{H}=R^{-1 / 2} H$. Applying orthogonal factorization to $\tilde{H}$, yields $\tilde{H}=Q U$. The update (3) is transformed to:

$$
\Delta x=U^{-1} \Delta \tilde{z},
$$

where: $\Delta \tilde{z}=Q^{\dagger} R^{-1 / 2}\left[z^{\text {meas }}-f(x)\right]$. Therefore the condition number of the transformed linear system is the condition number of matrix $U$, which is an upper triangular matrix. If in (1) we use the 2-norm [6]:

$$
\begin{aligned}
\text { Cond }\left(\tilde{H}^{\dagger} \tilde{H}\right) & =[\operatorname{Cond}(\tilde{H})]^{2}, \\
\operatorname{Cond}(U) & =\operatorname{Cond}(\tilde{H}) .
\end{aligned}
$$

Therefore:

$$
\operatorname{Cond}\left(M^{\prime}\right)=[\operatorname{Cond}(\tilde{H})]^{2},
$$

or,

$$
\operatorname{Cond}(U)=\left[\operatorname{Cond}\left(M^{\prime}\right)\right]^{1 / 2} .
$$

In Section III, we described that using 1-norm, $\operatorname{Cond}(M) \approx$ Cond $\left(M^{\prime}\right)$. Although (11) is developed using the 2-norm, from that we can qualitatively approximate $\operatorname{Cond}(U)$, using the 1-norm as:

$$
\operatorname{Cond}(U) \approx[\operatorname{Cond}(M)]^{1 / 2} .
$$

Therefore we can write:

$$
\begin{aligned}
C_{f v}^{U} & \approx\left[C_{f v}^{M}\right]^{1 / 2}, \\
C_{i f v}^{U} & \approx\left[C_{i f v}^{M}\right]^{1 / 2}, \\
C_{i v}^{U} & \approx\left[C_{i v}^{M}\right]^{1 / 2},
\end{aligned}
$$

where, superscript $U$ denotes the equivalent condition numbers for $U$. The estimates in (12)-(14), provide a better indication of the condition number of the linear systems solved in practical implementations compared to using the condition numbers of the information matrices evaluated in (7) and (10).

\section{RESULTS}

To make the development of the formulas described in Section IV possible, we have only considered radial networks. In practice networks are always looped. In this section we calculate the condition number of the information matrix of looped networks and compare the results with our developments using radial networks. To qualify our theoretical development describing the relative effects of different types and number of

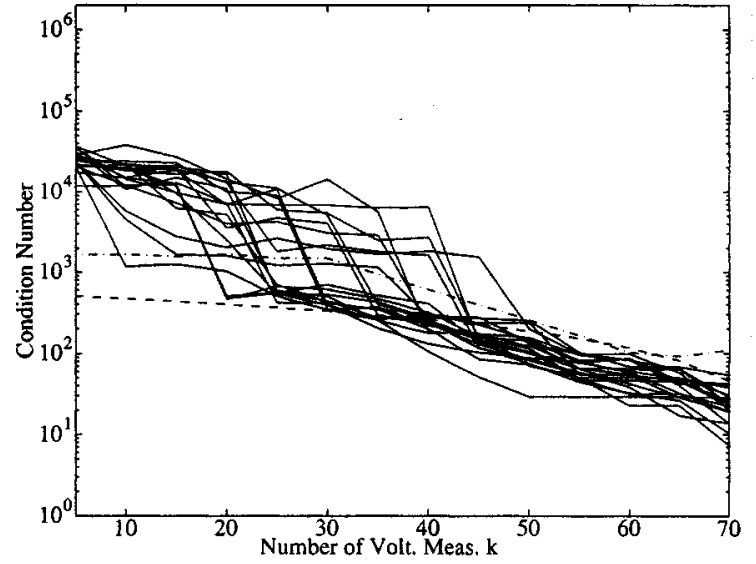

Fig. 3. The solid curves are condition numbers of $U$ for IEEE RTS-96 system with $n-k$ flow measurements and $k$ voltage measurements for 20 random placements of the measurements. The dot-dashed curve represents an ordered placement of the measurements. The dashed curve is the plot of the approximation in (12).

measurements, we present two case studies in Sections VI-A and VI-B. These cases are: the IEEE RTS-96 [11] and the IEEE 118 bus systems. These IEEE systems differ from our assumptions given in Section III in that they contain loops and have different admittances on different branches. Further, since practical state estimators consider a reference bus voltage angle, these cases contain a reference voltage angle. We assume a standard deviation of 0.02 for all branch power flow and bus injection measurement errors, and a standard deviation of 0.002 for all bus voltage magnitude measurement errors.

For most cases, we evaluated condition numbers for 21 different placements of measurements. The first, ordered placement, used the numbering of the buses and branches as provided in the data file to place the various measurements. Twenty others, random placements, were based on random permutations of bus and branch numbers. All condition numbers were calculated using the 1-norm.

\section{A. The IEEE RTS-96 System}

In this section we place measurements on the IEEE RTS-96 system [11] and calculate condition numbers for the matrix $U$. For the ordered placement, we place the flow measurements on the lowest numbered branches, voltage measurements on the buses following the last flow measurement, and injection measurements on the last series of buses following the last voltage measurement. Because of the nonradial configuration of the IEEE RTS-96 system, the placements of measurements according to the bus numbers affects the Jacobian matrix $H$ differently compared to the radial case. For the random placements, we use random permutations of the buses and branches.

Fig. 3 shows a plot of the condition number of $U$ using ordered and random placements of the measurements together with a plot of (12) for $k$ voltage measurements and $n-k$ flow measurements. These curves are all qualitatively similar. The condition number decreases as the number of voltage measurements increases and it increases as the number of flow measurements increases. When the number of voltage measurements is greater than about 40 , all of the curves are qualitatively similar. When there are less than 40 measurements, the curve with the 


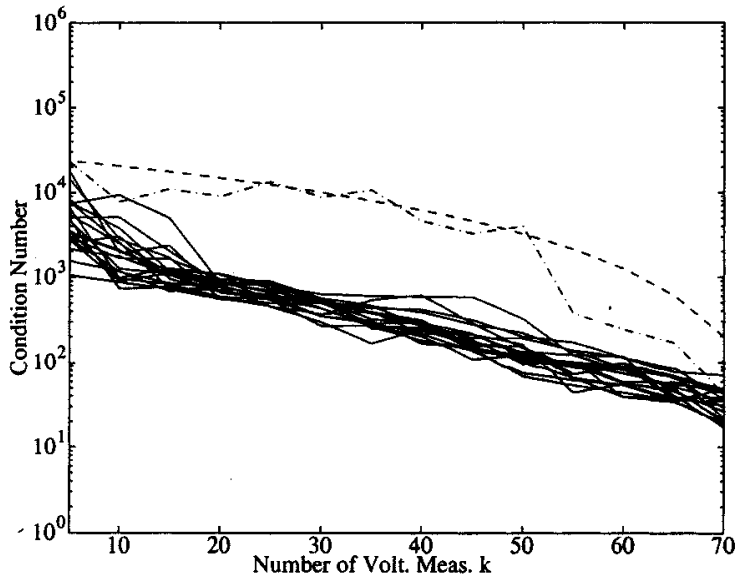

Fig. 4. The solid curves are condition numbers of $U$ for IEEE RTS-96 system with $n-k$ injection measurements and $k$ voltage measurements for 20 random placements of the measurements. The dot-dashed curve represents an ordered placement of the measurements. The dashed curve is the approximation in (14).

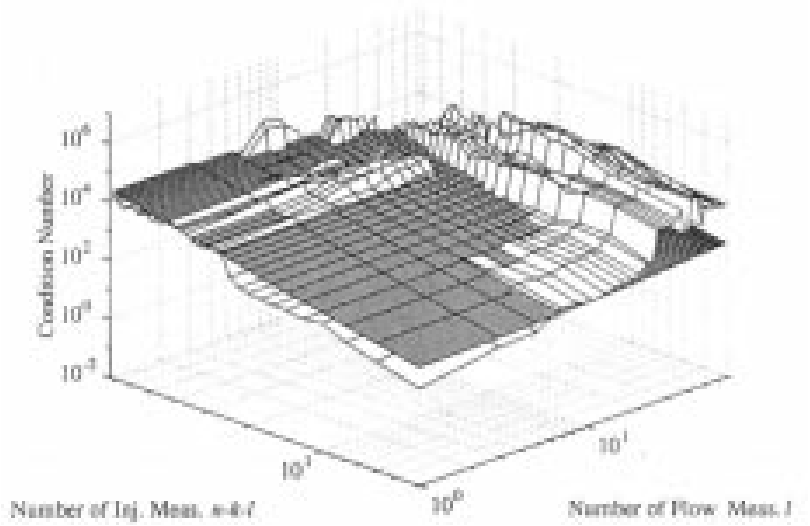

Fig. 5. The unshaded surface is condition number of $U$ for IEEE RTS-96 with $n-k-l$ injection measurements, $k$ voltage measurements, and $l$ flow measurements. The shaded plane is the plot of the approximation in (13).

ordered placement of measurements describing the condition number of $U$ follows the approximation in (12) more closely than the curves with the random placement of the measurements. Reference [5] shows that the effect of the branch reactance to resistance ratio on the condition number is very small. Thus we attribute the differences in magnitudes primarily to the nonradial topology of the IEEE RTS-96 system and the detailed placement of the measurements.

Fig. 4 shows a plot of the condition number of $U$ for the IEEE RTS-96 versus the number $k$ of voltage measurements, given $n-k$ injection measurements for ordered and random placements of the measurements, and also shows the approximation (14). Although the curve of the condition number of $U$ with ordered measurements contains some variations, it essentially matches the curve describing (14) to a large extent. The curves representing the random placements of the measurements are at lower magnitudes. The curve with the ordered placement of the measurements is closer to the approximation in (14). Nevertheless, the approximation in (14) is a reasonable guide to the qualitative dependence of condition number for all placements considered.

Fig. 5 shows a plot of the condition number of $U$ for the IEEE RTS-96 versus the numbers $n-k-l$ injection measurements,

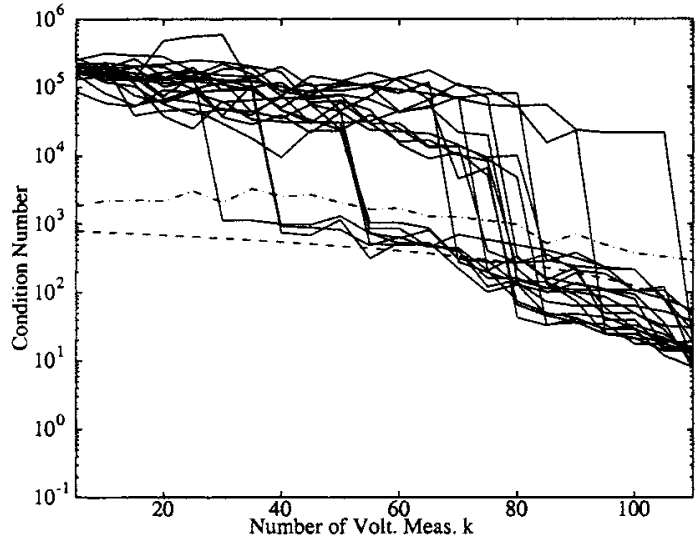

Fig. 6. The solid curves are condition numbers of $U$ for IEEE 118 bus system with $n-k$ flow measurements and $k$ voltage measurements for 20 random placements of the measurements and the dot-dashed curve represents an ordered placement of the measurements. The dashed curve is the plot of the approximation in (12).

and $l$ flow measurements, given $k$ voltage measurements for ordered placement of measurements. The surface describing the condition number of $U$ is the unshaded one, and cuts the plane of the approximation (13) in several locations. Overall (13) gives a good approximation to the magnitude of the condition number of $U$. The actual condition number is mostly higher than the approximation as the number of flow measurements increases and slightly lower as the number of injection measurements increases. When both the number of injections and flow measurements decrease, approximation (13) is slightly higher than the actual condition number of $U$. The variations and differences are predominantly attributed to the nonradial topology of the IEEE RTS-96 system, and the placement of the measurements.

\section{B. The IEEE 118-Bus System}

To further demonstrate the closeness of our formulas to the actual condition numbers as a function of the relative mixture of types and number of measurements, we apply similar measurements to the IEEE 118 bus system and calculate the condition numbers of $U$ and the corresponding approximations. Again, we place the flow measurements on the first series of branches, voltage measurements on the buses following the last flow measurement, and injection measurements on the last series of buses following the last voltage measurement.

Fig. 6 shows a plot of the condition number of $U$ for the IEEE 118 bus system versus the number $k$ of voltage measurements, given $n-k$ flow measurements for ordered and random placement of measurements. Although the plot of (12) and the condition number of $U$ match qualitatively, the condition number of $U$ is mostly at a higher magnitude for a nonradial topology. However, the condition number of $U$ for the IEEE 118 bus system follows the approximation (12) more closely for the ordered placement of the measurements. This may suggest that the closeness of the approximation to the actual condition number is a function of topology of the system.

Fig. 7 shows a plot of the condition number of $U$ for the IEEE 118 bus system versus the number $k$ of voltage measurements, given $n-k$ injection measurements for ordered and random 


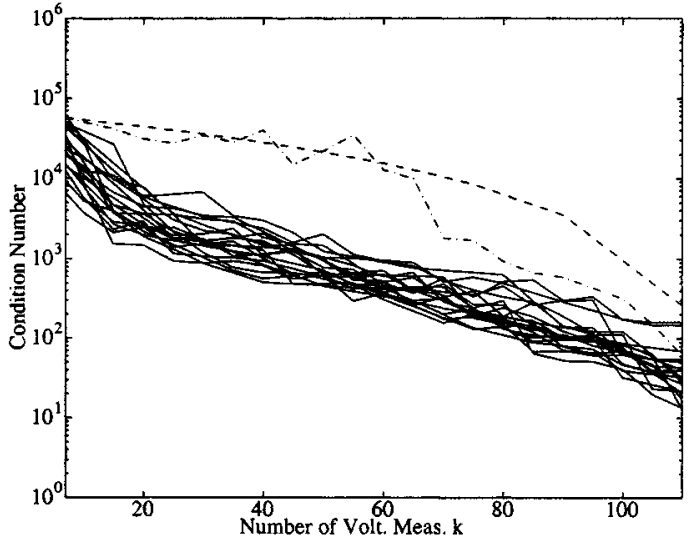

Fig. 7. The solid curves are condition numbers of $U$ for IEEE 118 bus system with $n-k$ injection measurements and $k$ voltage measurements for 20 random placements of the measurements and the dot-dashed curve represents an ordered placement of the measurements. The dashed curve is the plot of the approximation in (14).

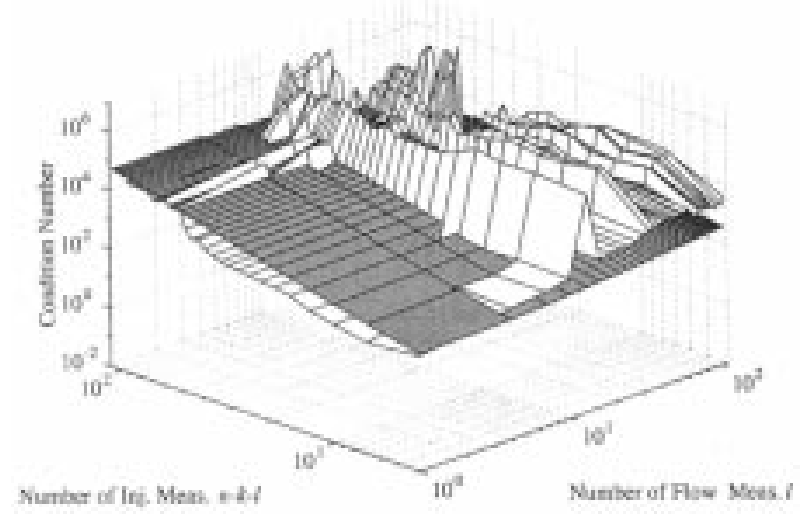

Fig. 8. The unshaded surface is condition number of $U$ for IEEE 118 bus system with $n-k-l$ injection measurements, $k$ voltage measurements, and $l$ flow measurements. The shaded plane is the plot of the approximation in (13).

placement of the measurements. The plot of (14) and the condition number of $U$ are close particularly for the ordered placement of the measurements and intersect close to the smaller number of flow measurements, with the plot of the condition number of $U$ being mostly at a lower magnitude than the plot of (14). It can be seen that as the number of voltage measurements increase the condition number rapidly decreases, and as the number of injection measurements increases the condition number increases.

Fig. 8 shows a plot of the condition number of $U$ and (13) for the IEEE 118 bus system versus the number $n-k-l$ of injection measurements and $l$ flow measurements, given $k$ voltage measurements for ordered placement of the measurements. The magnitudes of the condition number of $U$ matches the magnitudes described by the plane of the approximation (13). They are roughly at the same magnitude in most places.

\section{CONCLUSIONS AND FURTHER STUdieS}

This paper extends the results presented in [5] by developing formulas to describe the dependence of the condition number on the mixture of voltage, flow, and injection measurements.
Furthermore, it slightly generalizes the assumptions by introducing different measurement error variances for voltage and power measurements.

Besides the size of the system and variation of measurement error's standard deviations, the condition number of the state estimation information matrix and $U$ are predominantly a function of the placement, type, and number of measurements and network topology. In this paper we have investigated the relative effects of flow measurements in the presence of voltage and absence of injection measurements; injection measurements in the presence of voltage and absence of flow measurements, and injection measurements in the presence of flow and voltage measurements. We show that the presence of voltage measurements improves the condition number in the presence of flow or injection measurements, and the lower the proportion of the injection measurements in the presence of voltage and flow measurements the better the condition number of the information matrix. Since the state estimator solves for the voltage magnitudes and angles, increasing the proportion of these measurements always improves the condition number. Furthermore, the smaller the measurement error variances of the voltage and angle measurements the smaller the condition number of the information matrix.

The results of the examples presented in Section VI corroborate the formulas developed in Section IV, and demonstrate that the trends identified under restrictive assumptions, also qualitatively predict the trends for realistic systems. We suggest that the differences are predominantly the result of the system topologies and placement of the measurements. The approximation for the condition number of $U$ are generally closer for the ordered placements than the random placements of the measurements.

Comparing the IEEE RTS-96 system and the IEEE 118 bus system, the condition numbers for the IEEE RTS-96 system match more closely with the developed formulas of Section IV for the ordered placement of the measurements. We attribute this to the systems' topologies. Comparing the random placements with the ordered placements of the measurements, it appears that for the case of presence of injection and voltage measurements and absence of flow measurements, depicted in Figs. 4 and 7, the random placement of the measurements yields a similar trend that is always less than the approximation in (14). This may suggest that the order of placement employed for the development of (14) yields a maximum for condition number in the presence of voltage and injection measurements and absence of flow measurements. A future subject of research would be to describe the kind of variations in the topology and placement of measurements that would have significant effects on the condition number of the information matrix and $U$.

\section{REFERENCES}

[1] A. J. A. Simoes Costa and V. H. Quintana, "An orthogonal row processing algorithm for power sequential state estimation," IEEE Trans. on Power Apparatus and Systems, vol. 100, pp. 3791-3800, Aug. 1981.

[2] _ "Robust numerical technique for power system state estimation," IEEE Trans. on Power Apparatus and Systems, vol. 100, no. 2, pp. 69-698, Feb. 1981.

[3] R. Ebrahimian, "Power system operations: State estimation distributed processing," Ph.D. dissertation, University of Texas at Austin, Austin, 1999. 
[4] M. E. El-Hawary, Electrical Power Systems Design and Analysis. Piscataway, NJ: IEEE Press, 1995.

[5] J. W. Gu, G. R. Krumpholz, K. A. Clements, and P. W. Davis, "The solution of ill-conditioned power system state estimation problems via the method of Peters and Wilkinson," IEEE Trans. on Power Apparatus and Systems, vol. PAS-102, no. 10, pp. 3473-3480, Oct. 1983.

[6] S. G. Nash and A. Sofer, Linear and Nonlinear Programming, 2nd ed. New York, NY: Wiley, 1996.

[7] E. W. Palmer and G. Ledwich, "Optimal placement of angle transducers in power systems," IEEE Trans. on Power Systems, vol. 11, no. 2, pp 788-793, May 1995.

[8] A. G. Phadke, "Synchronized phasor measurements in power systems," IEEE Computer Applications in Power, vol. 6, no. 2, pp. 10-15, Apr. 1993.

[9] R. C. Pires, A. S. Costa, and L. Mili, "Iteratively reweighted least-squares state estimation through Givens rotations," IEEE Trans. on Power Systems, June 1998.

[10] W. H. Press, B. P. Flannery, S. A. Teukolsky, and W. T. Vetterling, $N u$ merical Recipes The Art of Scientific Computing. New York, NY: Press Syndicate of the University of Cambridge, 1989.

[11] Reliability Test System Task Force of the application of probability methods subcommittee, "The IEEE reliability test system-1996," in IEEE/PES Winter Meeting, Baltimore, MD, Jan. 21-25, 1996, 96 WM 326-9 PWRS
[12] B. Stott and O. Alsac, "Fast decoupled load flow," IEEE Trans. on Power Apparatus and Systems, vol. PAS-93, no. 1, pp. 859-869, May/June 1974.

[13] N. Vempati, I. W. Slutsker, and W. F. Tinney, "Enhancements to Givens rotations for power system state estimation," IEEE Trans. on Power Systems, vol. 6, no. 2, pp. 842-849, May 1991.

[14] A. J. Wood and B. F. Wollenberg, Power Generation, Operation, and Control, 2nd ed. New York, NY: Wiley, 1996.

Reza Ebrahimian received the B.S. and Masters in electrical engineering from Texas A\&M University, and the Ph.D. degree from the University of Texas at Austin. He is currently a Consulting Engineer at Austin Energy.

Ross Baldick received the B.Sc. and B.E. degrees from the University of Sydney, Australia and the M.S. and Ph.D. degrees from the University of California, Berkeley. He is currently an Associate Professor in the Department of Electrical and Computer Engineering at the University of Texas at Austin. 